\title{
Las representaciones sociales de la educación ambiental en clases de educación física, de los estudiantes y profesores de establecimientos educacionales de la IX región de la Araucanía, Chile \\ Social representations of environmental education in physical education classes of students and teachers of educational establishments of the IX region of Araucanía, Chile

\author{
Carlos Roberto Arriagada Hernández
} \\ Universidad Autónoma de Chile (Chile)
}

\begin{abstract}
Resumen. La Educación Ambiental como contenido está vinculado de manera incipiente en el currículum nacional, la cual, por el contexto actual global y regional debería ser un eje central y transversal, que persiga concientizar, formar y modificar actitudes sobre el cuidado del ambiente desde las edades tempranas y durante toda la escolaridad. El propósito de la investigación es describir y comprender en profundidad las representaciones sociales de los estudiantes y profesores de Educación Física sobre Educación Ambiental de los establecimientos educacionales de las comunas Curacautín, Temuco y Puerto Saavedra de la IX Región de la Araucanía. La metodología de esta investigación es de enfoque cualitativo, la cual se lleva a cabo a través de entrevistas semiestructuradas abiertas, los datos son recopilados y analizados a través del software Atlas.ti versión 8. Los principales resultados de este estudio evidencian el escaso conocimiento de los estudiantes en relación con la Educación Ambiental. De igual forma los docentes de cada establecimiento consienten integrar la Educación Ambiental dentro de sus clases, pero de forma secundaria, sin abarcar contenidos concretos directamente relacionados con el cuidado del medio ambiente. Finalmente se concluye, que las representaciones sociales de los docentes y estudiantes revelan un interés por el tema y expresan diversas opiniones e ideas respecto de cómo podría ser abordado el contenido, de manera que el conocimiento sobre EA crezca y se desarrolle en el ámbito escolar.

Palabras clave: Educación Ambiental; Educación Física; Desarrollo Integral; Escolares, Profesores.
\end{abstract}

\begin{abstract}
Environmental Education as a content is incipiently linked to the national curriculum, which, due to the current global and regional context, should be a central and transversal axis that seeks to raise awareness, train and modify attitudes on environmental care from early ages and throughout schooling. The purpose of the research is to describe and understand in depth the social representations of Physical Education students and teachers about Environmental Education in educational establishments in the communes of Curacautín, Temuco and Puerto Saavedra in the IX Region of Araucanía. The methodology of this research is of qualitative approach, which is carried out through open semistructured interviews, the data are collected and analyzed through the Atlas.ti version 8 software. The main results of this study show the scarce knowledge of the students in relation to Environmental Education. Likewise, the teachers of each establishment agree to integrate Environmental Education in their classes, but in a secondary way, without including concrete contents directly related to the care of the environment. Finally, it is concluded that the social representations of teachers and students reveal an interest in the subject and express diverse opinions and ideas regarding how the content could be approached, so that knowledge about EE grows and develops in the school environment.
\end{abstract}

Keywords: Environmental Education; Physical Education; Integral Development; Schoolchildren, Teachers.

\section{Introducción}

A nivel mundial y a lo largo de Chile, la contaminación se ha situado como una problemática constante, así como el poco conocimiento sobre Educación ambiental y la poca conciencia en este tema, que indudablemente involucra y afecta a todas las personas. Según conclusiones del informe de Perspectivas del medio ambiente mundial, la humanidad no está en condiciones de cumplir las metas propuestas para el 2030 y 2050 mencionados en los distintos acuerdos internacionales sobre cambio climático, desarrollo sostenible y protección medioambiental. Se requieren medidas urgentes aho-

Fecha recepción: 22-07-21. Fecha de aceptación: 20-11-21

Carlos Roberto Arriagada

carlosarriagadah@gmail.com ra, sostiene la ONU (Gómez-Lee, 2019). Torres, Benavides, Latoja y Novoa (2017) mencionan que, de acuerdo con lo establecido por la ONU, se quiere lograr formar una población mundial que tenga conciencia y se interese por el medio ambiente y por los problemas relacionados a la contaminación. Por lo que en Chile se crean nuevas leyes e instituciones que ayudan a lograr las metas propuestas a nivel país.

De acuerdo con el Ministerio del Medio Ambiente (2018) en Chile, la Ley 19.300 señala que la Educación Ambiental es «un proceso permanente de carácter interdisciplinario destinado a la formación de una ciudadanía que reconozca valores, aclare conceptos y desarrolle las habilidades y las actitudes necesarias para una convivencia armónica entre seres humanos, su cultura y su medio físico circundante» (p.27). Lo que nos da a entender que lo definen como un proceso que se 
debiese desarrollar durante toda la vida, convirtiéndolo en un hábito no solo para el beneficio personal, sino que además para llevar una buena convivencia con las demás personas.

La Educación Ambiental va de la mano del desarrollo sustentable, lo que, para el Ministerio de Educación (2013) significa:

formar niñas, niños, adolescentes, jóvenes y adultos, todos actores de la comunidad educativa, para fortalecer aquellos conocimientos, habilidades y actitudes que les permitan tomar decisiones conscientes y responsables frente a las problemáticas sociales, ambientales, culturales y económicas, y que estas tienen consecuencias, positivas o negativas, que los implican, así como a la familia, al barrio, la escuela o el jardín infantil, la comuna o el país. (p.5)

El interés de investigar las representaciones sociales (Moscovici, 2012) de los estudiantes y profesores emerge mediante la necesidad de buscar un cambio de actitud en las personas y por sobre todo en las nuevas generaciones, concientizar a los jóvenes y a la comunidad en general sobre la importancia del cuidado y preservación del entorno, mismo entorno que es compartido para coexistir con otras personas y que representa un aspecto fundamental en la calidad de vida de las personas (Pulido y Olivera, 2018). Además, con la teoría de las representaciones sociales da relevancia a las formas de conocimiento popular y sentido común que son compartidos e integradores de la construcción de la realidad social (Berger y Luckmann, 1999, citado por Diaz, 2020) en este caso sus perspectivas en formación de la educación ambiental. Las representaciones sociales son un proceso de reconstrucción, que modifican y recrean activamente el fenómeno de investigación (Moscovici, 1988). Las representaciones surgen a través de proposiciones, valoraciones y creencias de los grupos sociales, clasificándolas en tres dimensiones, actitud, información y campo de representación. La primera como representación global respecto de un objeto, la segunda, la organización de conocimientos respecto de un fenómeno y la tercera es la jerarquización de los elementos que componen la representación, como contenido concreto (Moscovici, 1979).

En cuanto a la Educación Física y la Educación Ambiental (en adelante, EA) Corrales, (2010), señalan «la Educación Física no solo debe tratar la EA dentro del ámbito educativo, sino que posee una situación privilegiada para su desarrollo, tanto por los contenidos que le son propios como por ser una de las escasas asignaturas basadas principalmente en las experiencias prácticas y las vivencias personales» (pp.46-47).

Algunos autores hacen referencia del rol del profesor debido a que es el mediador entre lo que se diseña en el currículum y el aprendizaje al igual que en la adquisición de conciencia ambiental por parte de los estudiantes (Sureda-Negrete, Oliver-Trobat, CatalanFernandez y Comas-Forgas, 2014). Wilke (1995, citado por Torres, Benavides, Latoja \& Novoa, 2017) menciona que es importante contar con un profesor que tenga los conocimientos necesarios para el desarrollo de habilidades y la formación de valores, que tenga conocimientos y actitudes ambientales, lo que generará estudiantes más consientes con el medio ambiente (Torres et al, 2017).

Esta investigación busca inspirar a centros educacionales, conociendo la perspectiva de sus estudiantes y las falencias que se deban mejorar, a la sociedad en crear conciencia en las nuevas generaciones y además a profesores de Educación Física, generando conocimiento de la educación ambiental para identificar problemas y plantear soluciones que contribuyan al cuidado de la naturaleza (Yeh, Ma \& Huan, 2016).

La metodología de esta investigación es de enfoque cualitativo, la cual se llevará a cabo a través de entrevistas semiestructuradas abiertas y los datos serán recopilados y analizados a través del software Atlas.ti versión 8, del cual se extraen redes semánticas a partir de las codificaciones en base a los datos.

En consecuencia, el propósito del presente trabajo fue: describir y comprender en profundidad las representaciones sociales de los estudiantes y profesores de Educación Física sobre Educación Ambiental de los colegios Armando Dufey y Greenhouse de la comuna de Temuco.

Asimismo, en esta investigación respecto de la Educación Ambiental para los estudiantes y profesores, como principales conclusiones se dilucida como se manifiesta en las vidas de estudiantes y profesores, como es su aporte desde el contexto escolar, y el conocimiento de estrategias de como abarcan los profesores el contenido de éste, y sobre todo durante las clases de educación física.

\section{Material y Método}

\section{Tipo de investigación}

La investigación es de enfoque cualitativo, su diseño corresponde a la Teoría Fundamentada esta como «método permite al investigador poder construir una teoría sustantiva que permita interpretar la subjetividad, 
ideología, percepción, constructos, discursos, etc. que construyen los individuos que se encuentran en el estudio» (Cresswell, 2014, p.15). Para el proceso de investigación la Teoría Fundamentada, los datos se categorizan con codificación abierta, luego el investigador organiza las categorías resultantes en un modelo de interrelaciones (codificación axial), que representa a la teoría emergente y explica el proceso o fenómeno de estudio (codificación selectiva) (Straus \& Corbin, 2002; Gibbs, 2012; Stake, 2015).

\section{Participantes}

La población que fue seleccionada para esta investigación corresponde a estudiantes de sexto año básico y profesores de Educación Física de los establecimientos San Andrés, Greenhouse, Armando Dufey y Piedra Alta de las comunas de Curacautín, Temuco y Puerto Saavedra. Los participantes correspondientes fueron seis profesores de Educación Física de enseñanza básica y 14 estudiantes de sexto año básico de dichos establecimientos (Tabla 1 y 2).

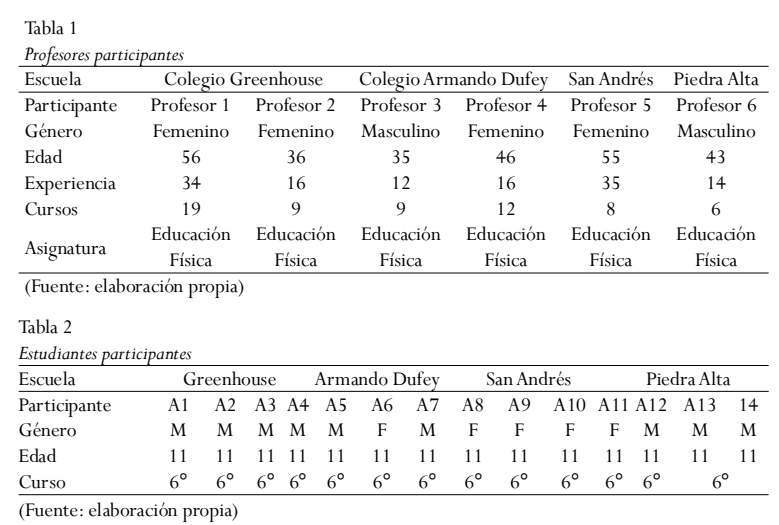

\section{Dimensión ética del estudio}

En este aspecto las identidades reales de cada uno de los participantes del estudio fueron resguardadas bajo absoluto anonimato, al igual que las respuestas reveladas en las entrevistas. Solo las entrevistadoras tuvieron conocimiento de las identidades de los participantes implicados en el estudio y no se dan a conocer en los resultados ni en la extensión de éste.

\section{Instrumentos}

El tipo de instrumento que se utilizó en esta investigación son entrevistas semiestructuradas abierta (Bryman, 2016).

Según Taylor \& Bogdan (1987), en este tipo de instrumento se logra una comunicación y la construcción conjunta de significados respecto a un tema.

Las entrevistas semiestructuradas, utilizan pregun- tas que guían la conversación y el entrevistador tiene libertad de introducir preguntas adicionales para precisar conceptos u obtener más información sobre el fenómeno en investigación (Bryman, 2016).

Según lo planteado anteriormente, es que esta investigación se realizó con dos tipos de entrevistas, una dirigida a estudiantes y otra a profesores (a), las cuales contaron con siete preguntas respectivamente.

En relación con la creación del instrumento, fue validado bajo el método de Delphi (Bryman, 2016) y este se divide en cuatro categorías relacionada con los objetivos específicos del estudio(Tabla 3). Cada categoría, contiene una cantidad de preguntas para ser realizadas al momento de la entrevista y rescatar las observaciones de los participantes en cuanto a la opinión sobre los distintos temas expuestos, con la finalidad de crear un ambiente propicio el cual entregue la confianza para realizar una entrevista o conversación guiada, la cual hará al participante expresarse sobre el tema de la investigación.

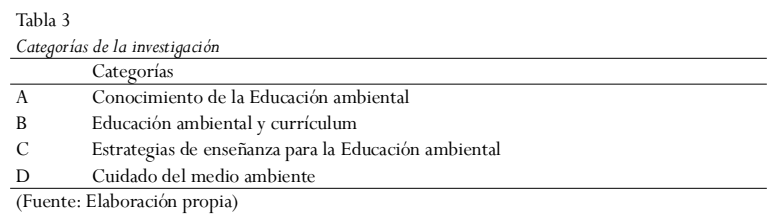

\section{Análisis de los datos}

Los datos recopilados fueron analizados a través del software Atlas.ti versión 8, del cual se extrajeron redes semánticas a partir de las codificaciones de las entrevistas, documentos, artículos científicos, entre otros. Atlas.ti es un programa que permite trazar redes conceptuales para facilitar la elaboración de modelos teóricos, y escritura de los hallazgos (Arriagada \& Fuentes, 2021). Atlas.ti es el principal soporte informático para desarrollar teoría fundamentada, este programa fue diseñado a finales de los ochenta por el alemán Thomas Murh (San Martin, 2014). Este método de análisis aumenta la calidad de la investigación educativa, puesto que fortalece la coherencia y el rigor de los procedimientos analíticos (Arriagada \& Fuentes, 2021). Principalmente, se reconocen como ventajas la rapidez que otorga a procesos mecánicos como: segmentación, recuperación y codificación de información (Amezcua \& Gálvez, 2002).

Atlas.ti es un programa de recuperación de texto, sin embargo, está dirigido a un trabajo conceptual, en donde cada paso de la codificación teórica (codificación abierta, axial y selectiva) tiene un espacio en el programa (San Martin, 2014). Por lo que este programa facili- 
ta la organización del análisis a través de funciones que permiten: segmentar citas, conceptualizar, registrar reflexiones, categorizar, relacionar procesos y mostrar la teoría que se construye a través de diagramas (San Martin, 2014).

\section{Resultados}

A continuación se presentan las principales categorías y subcategorías del análisis de las entrevistas realizadas a docentes y estudiantes (Tabla 4).

\begin{tabular}{cl}
$\begin{array}{l}\text { Tabla } 4 \\
\text { Categorías y subcategorías relevantes de los participantes entrevistados de la investigación. }\end{array}$ \\
\hline \multicolumn{1}{c}{ Categoría } & \multicolumn{1}{c}{ Subcategorías } \\
\hline & Conservación de la naturaleza \\
& Desarrollo sustentable \\
Educación ambiental & Conservación de la naturaleza \\
& Contaminación \\
& Ed Física importante \\
& Conciencia por la educación ambiental \\
\hline & Planes y programas \\
& Bajo desarrollo de la EA en EF \\
& Medio natural \\
& EA transversal \\
& Desconocimiento de los contenidos de EA \\
& Actividad física y motricidad \\
\hline Contenidos curriculares & Interacción con el medio natural \\
& Proyectos \\
& Estrategias de autocuidado \\
& Formación de hábitos \\
& Actividades en la naturaleza \\
Estrategias de enseñanza & Desconocimiento de estrategias \\
\hline (Fuente: Elaboración propia) &
\end{tabular}

Categoría: Profesores/as y estudiantes de establecimientos, cconocimiento de la Educación Ambiental (figura 1).

En relación con el conocimiento de la EA, los docentes representan dos conceptos claves en relación con las preguntas realizadas. En primer lugar, reconocen la existencia de la EA, la importancia de esta y la conciencia que se debe desarrollar desde la asignatura de Educación Física y salud en los estudiantes para el cuidado del medio ambiente. Esta importancia se atribuye a la representación social que simbolizan los docentes en relación con el deterioro del medio ambiente, lo cual provoca la necesidad de generar escuelas que desarrollen este tipo de temáticas a nivel curricular y de formación integral.
E3: «La educación ambiental es un proceso de formación que desarrolla actitudes, valores y habilidades para una integral relación entre las personas y su medio ambiente».

E2: "La importancia radica en la necesidad de formar futuras generaciones con conciencia y responsabilidad en el cuidado de la naturaleza, capaces de asumir los desafíos del calentamiento global, deterioro del aire, extinción de especies, entre otros sucesos negativos causados principalmente por la acción humana».

E3: «Es de suma importancia la educación ambiental, pues es un tema transversal a todos los niveles educacionales y a todas las asignaturas, en el cual puede ser abordado desde lo actitudinal, procurando una toma de conciencia, un cambio de actitud, que favorezca la sana convivencia con nuestro medio ambiente para conservar y mejorar nuestra calidad de vida».

En relación con el conocimiento de la EA, los estudiantes mencionan la importancia de tener conciencia por la EA para la conservación y cuidado de la naturaleza, al igual que el cuidado de los distintos ambientes en donde ellos se desarrollan día a día al aire libre, en específico en la limpieza, evitando botar papeles para no contaminar, no obstante, mencionan tener poco conocimiento sobre los contenidos de la EA y de las distintas formas para el cuidado de este. Esto quiere decir que, desde el contenido o información, los estudiantes representan desde sus experiencias dimensiones simbólicas que caracterizan el conocimiento de la Educación ambiental asociándose a una valoración positiva o negativa con relación este fenómeno desde una perspectiva social.

E7: «Para mí la Educación Ambiental es aprender a cuidar el planeta.»

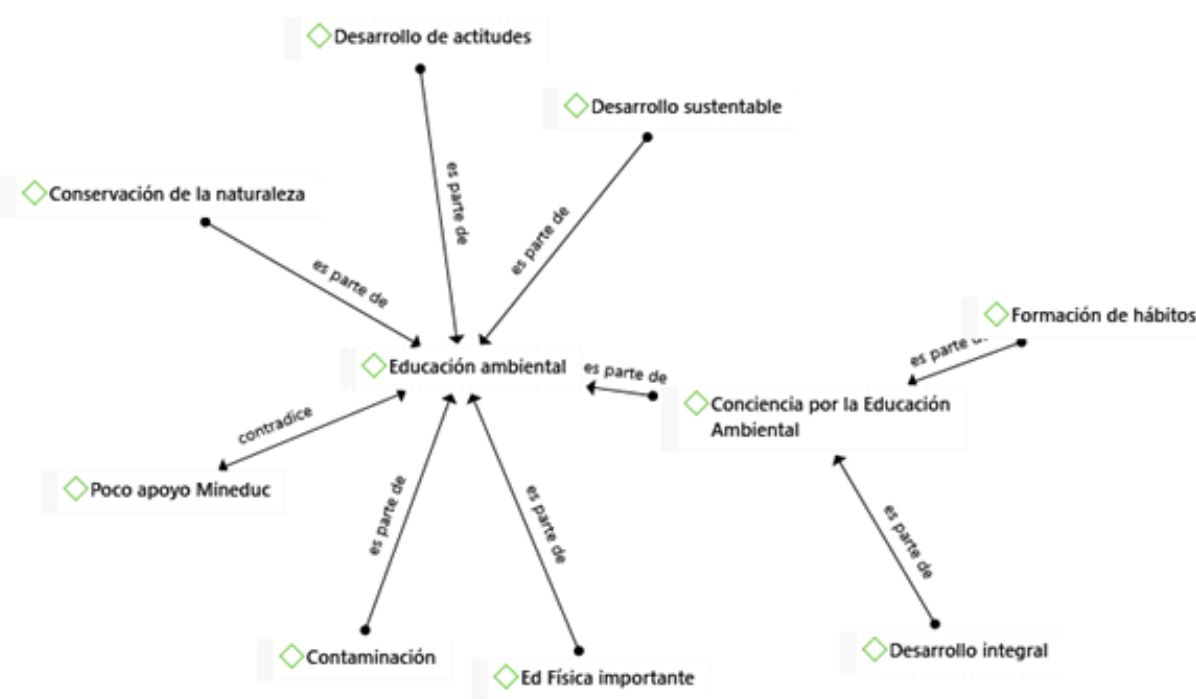

Figura 1. Red semóntica $N^{\circ} 1$, Conocimiento de la Educación Ambiental (Fuente: elaboración propia con utilización de Atlas.ti) 
E5: "No sé mucho sobre esto, pero para mí es la Educación sobre el ambiente.»

E7: «Si es importante porque debemos cuidar al planeta y aprendemos a no botar basura»

En relación con la interpretación de los resultados de esta categoría, Delors (1996) menciona, «la educación a lo largo de la vida se basa en cuatro pilares: aprender a conocer, aprender a hacer, aprender a vivir juntos, aprender a ser»(p.34), en donde además la UNESCO (2006) reafirmo esta propuesta y agregó aprender a transformarse a uno mismo y a cambiar la sociedad. Esto quiere decir que el conocer es la base de los aprendizajes, en donde el aprender desde la experiencia aporta a lograr un sello personal, respetar a los demás y generar cambios en la sociedad.

Mora (2013) expresa que la base del conocimiento de los estudiantes sobre la EA es determinante para las nuevas generaciones, para que sean conscientes, comprendan y contribuyan con acciones que permitan direccionar un futuro sostenible.

Estos autores afirman que las nuevas generaciones son determinantes para el futuro, por lo que es importante que estos tengan conocimiento y conciencia sobre la importancia del cuidado y conservación del ambiente.

En este sentido la EA es el resultado de diversas disciplinas y experiencias que facilita la percepción integrada de los problemas del medio ambiente, haciendo posible una acción más racional y capaz de responder a las necesidades sociales.

En Chile, la Ley 19.300 de Bases del Medio Ambiente la define como un proceso permanente de carácter interdisciplinario, al igual que Corrales (2010) en su estudio trabajar la EA desde la Educación física en donde menciona, que la EA es un proceso continuo, que busca formar y modificar las actitudes de las personas.

Desde las representaciones sociales, las categorías se relacionan desde una perspectiva simbólica en la cual los docentes y estudiantes, desde sus experiencias y vivencias, brindan un significado utilizando el lenguaje para socializar dicho conocimiento, atribuyendo características positivas o negativas del fenómeno estudiado.

Categoría: Profesores y estudiantes de los establecimientos,

\section{Educación Ambiental y currículum (figura 2).}

En relación al currículum nacional, los profesores mencionan la EA como un objetivo transversal en los planes y programas de Educación Física, en donde la actividad física y la motricidad son fundamentales para el desarrollo de habilidades y el desarrollo integral de los estudiantes, no obstante, afirman tener un desconocimiento sobre los contenidos de la EA, lo que genera un bajo desarrollo de ésta temática en las clases de Educación Física, además del poco apoyo por parte del Mineduc para generar contenidos y sugerencias en momentos de crisis o restricción ambiental.

E1: «Es importante abarcar la educación ambiental en la asignatura de educación física, especialmente en los objetivos fundamentales que tienen relación con la actividad física y motriz en el entorno natural.»

E2: «Claro que es importante, ya que en nuestros planes y programas se trabaja con el entorno natural, en base a ello poder hacer actividades con objetivos de cuidar nuestro entorno aceptando las normas del medio urbano y natural.»

E3: "En cuanto a las clases de educación física, se ve reflejado en las medidas aplicadas cuando se produce la restricción en el período de crisis o contaminación ambiental, estas medidas refieren a evitar las clases y /o actividad fisca en espacios abier-

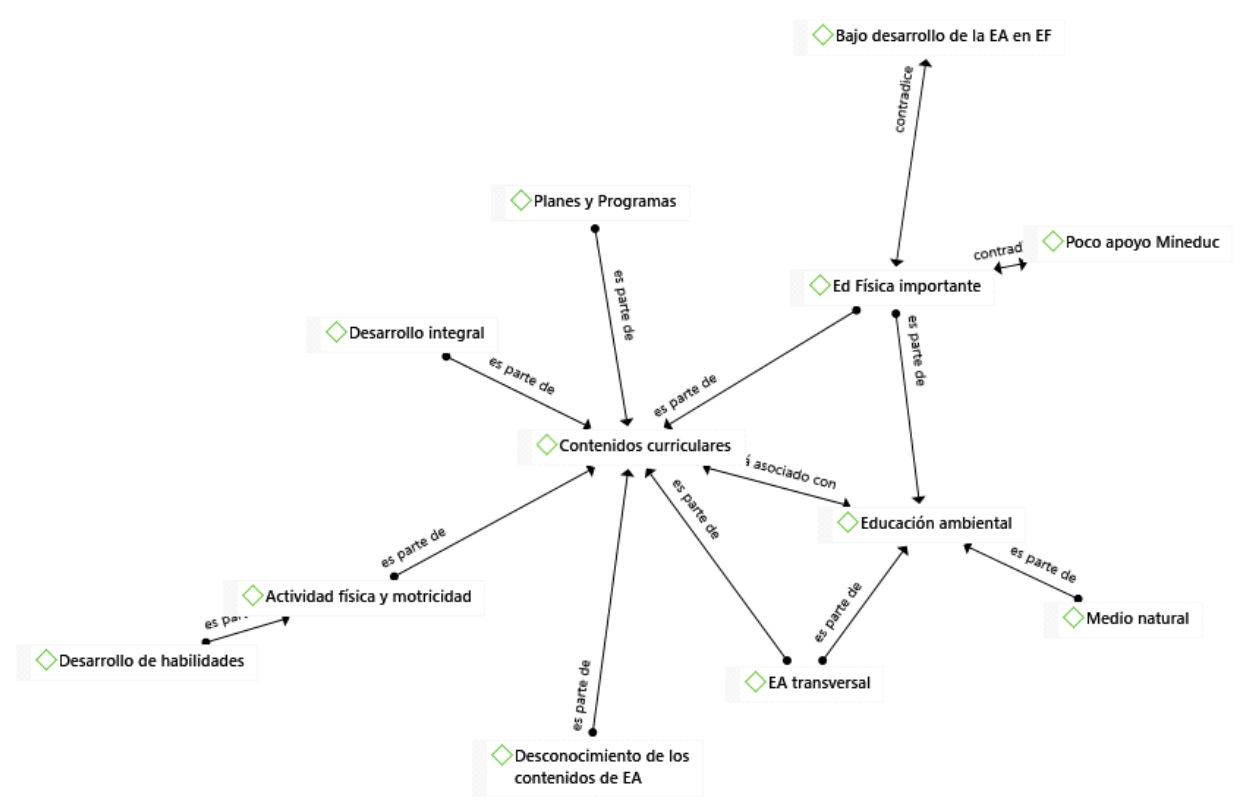

Figura 2 . Red semántica $N^{\circ}$ 2, Educación Ambiental y currículum

(Fuente: elaboración propia con utilización de Atlas.ti) 
tos, pero no sugieren actividades o no aportan con actividades y/o material adecuado para implementar y aplicar en casos de no poder realizar las clases, lo que podría ser un apoyo importante en la educación ambiental a la que nos referimos."

Según lo planteado por el Ministerio del Medio Ambiente (2018), «Chile tiene un currículum prescriptivo, donde los aprendizajes se implementan a través de conocimientos, habilidades y actitudes» (p.54).

El objetivo de aprendizaje número 4 del programa de estudio propuestos por el Ministerio del Medio Ambiente (2018) (en adelante, MMA) de $6^{\circ}$ Año básico en la asignatura de Educación Física, menciona lo siguiente: «Planificar y ejecutar actividades físicas y deportivas, utilizando diversos entornos y aplicando medidas para conservar limpios y ordenados los espacios; por ejemplo: planificar un campamento, incluyendo actividades deportivas» (p.6).

No se presenta un objetivo de aprendizaje en específico del cuidado, mantención y prevención del medio ambiente como contenido, sino más bien como un objetivo transversal y actitudinal. Por lo que no se puede asegurar que durante el proceso educativo los docentes realicen clases destinada a la EA.

En Chile, se promulgó el año 2016 la Ley 20.911, que mandata la elaboración de un Plan de Formación Ciudadana, en todos los establecimientos educacionales reconocidos por el Estado, en los niveles de enseñanza parvularia, básica y media (MMA, 2018, p.45). Y que este plan es definido como:

Es un instrumento de planificación (con objetivos, acciones, plazos y responsables) que debe permitir a las comunidades educativas promover la mejora permanente de todos los aprendizajes que involucra la formación ciudadana mediante espacios académicos, formativos, culturales y extracurriculares, con el fin de desarrollar en los estudiantes y en toda la comunidad educativa, las actitudes y habilidades necesarias para participar de manera constructiva y democrática en la sociedad actual.

Este plan pertenece a los planes obligatorios por normativa, y en este plan en particular debe tener a lo menos una acción que se vincule con la EA para la formación de hábitos, conciencia y actitudes para desarrollar futuros ciudadanos que tengan incorporado este elemento fundamental debido a los actuales problemas del siglo XXI como el calentamiento global, contaminación y desarrollo sustentable.

De acuerdo con el currículum nacional por parte de los estudiantes, se interpreta que la EA logra generar en ellos el desarrollo de distintas actitudes positivas para el cuidado de la naturaleza, además de la formación de hábitos, para que así, puedan tener un trato adecuado relacionado al entorno que nos rodea. Sin embargo, dando un enfoque al ámbito de la Educación Física, se plantea la poca presencia y bajo desarrollo de la EA en la asignatura. A pesar de esto, existen diversas actividades que realizan, pero no de manera regular, como, por ejemplo: cicletadas, salidas a parques, senderismo o simplemente desarrollar la clase fuera del aula en un ambiente natural.

E8: "No realizan actividades de ese tipo en mi colegio»

E9: «No hacemos actividades, pero siempre se preocupan de cuidar el medioambiente en el colegio»

E6: «Muy poco, cuando salimos a caminar, o cuando salimos al parque estadio y lo limpiamos o cuando jugamos al aire libre»

Si bien se enseña en los establecimientos a cuidar la naturaleza y medioambiente, se queda al debe en relación con actividades que se enfoquen en la EA.

Se tiene la convicción de que la Educación Física no sólo debe tratar la EA dentro del ámbito educativo, sino que posee una situación privilegiada para su desarrollo, tanto por los contenidos que le son propios, como por ser una de las escasas asignaturas basadas principalmente en las experiencias prácticas y las vivencias personales (López \& López, 1997, p. 79).

López \& López, (1997) menciona que parece bastante lógico pensar que la realización de actividad física posee una serie de ventajas, como toma de contacto con la naturaleza, eliminación del estrés, mantenimiento de la forma física y un sinfín de características positivas para el ser humano, las cuales la mayoría pueden constituir razones más que suficientes para la inclusión de estas actividades relacionadas a la EA en el ámbito escolar.

Se interpreta por medio de estos autores que es importante que exista una conexión entre la EA y el ámbito escolar, más específicamente en la Educación Física. Unir ambos conceptos de manera que se puedan realizar actividades relacionadas entre sí es de utilidad para los niños y niñas, ya que de esta manera abarcan conocimiento sobre el cuidado del medioambiente además de practicar actividad física.

Desde las representaciones sociales, los docentes y estudiantes representan la educación ambiental y el currículum como importante, y se da en relación desde lo individual a lo grupal, visualizando la necesidad de desarrollar actividades en relación a la EA y conocerla en mayor profundidad. 
Categoría: Profesores de los establecimientos educacionales, Estrategias de enseñanza para la Educación Ambiental (figura 3).

Los docentes manifiestan tener un desconocimiento de estrategias para la EA en sus clases, sin embargo, mencionan algunas como, por ejemplo: estrategias de cuidado y autocuidado, proyectos y cicletadas. En este tipo de actividades los docentes promueven el cuidado y preservación del medio ambiente, a través de la entrega de conocimientos y actitudes que aseguren una conducta positiva y favorable de parte de los alumnos con el medio ambiente, que sean aplicables al diario vivir. Asimismo, los docentes coinciden en que estas medidas son tomadas en base a apreciaciones más bien personales o indicaciones del colegio, respecto de cómo creen que pueden abordar el contenido de mejor manera y no precisamente bajo la orientación del Ministerio de Educación u otra entidad en particular.

E2: «Realizando actividades en el medio natural, aceptación y normas con respecto al medio ambiente, actividad física en el entorno protegiendo el área en el cual se hace la actividad, ejemplo hacer senderos, trote en el medio natural, actividades que protejan y cuiden nuestro medio».

E1: "actividad física en el entorno protegiendo el área en el cual se hace la actividad, ejemplo hacer senderos, trote en el medio natural, actividades que protejan y cuiden nuestro medio».
La Educación Física al aire libre se centra en el conjunto de conocimientos, habilidades, destrezas, técnicas y recursos que permiten desenvolverse o practicar actividades físicas lúdico-deportivas en la naturaleza, con seguridad y con el máximo respeto hacia su conservación; disfrutando, compartiendo y educándose en ella (Baena \& Granero, 2008).

En relación con lo anterior, se puede concluir que los docentes entregan conocimientos sobre EA a sus alumnos, pero siempre tras los objetivos que esperan cumplir en la asignatura, es decir, a la hora de realizar una clase el objetivo principal de ésta no es centrarse específicamente en los conocimientos de EA, cualesquiera sean estos, sino en cumplir con el objetivo de esa clase en particular y a ésta sumar algunas prácticas o conocimientos de EA, lo que no es el escenario ideal si lo que se busca es que los alumnos vayan creciendo en cuanto al entendimiento y cambios de conducta respecto del tema.

E3: «En nuestro establecimiento educacional, y en nuestras clases de educación física no utilizamos métodos nuevos establecidos por el ministerio de educación, siempre mantenemos y fortalecemos conductas de higiene, orden y aseo en el espacio a trabajar».

En chile la EA se presenta de manera transversal en el currículum escolar. Sin embargo, que esta se desarrolle como tal o que se tome en cuenta en los diseños escolares, depende posiblemente de la formación de los profesionales de la educación en esta área y del tratamiento que se le da en su proceso de formación inicial (CastilloRetamal \& CorderoTapia, 2019).

Respecto a lo anterior, se puede afirmar que los docentes más que enseñar nuevas prácticas, conocimientos o aplicar nuevos métodos para el desarrollo de la EA en el aula, fortalecen los conocimientos que ya están presentes o desarrollan solamente algunos hábitos vinculados a la

Figura 3. Red semántica $N^{\circ} 3$, Estrategias de enseñanza para la Educación Ambiental (Fuente: elaboración propia con utilización de Atlas.ti) 
EA desde una mirada tradicional con baja disposición al cambio, lo que puede resultar redundante y poco llamativo para los estudiantes. Frente a este escenario, los docentes tampoco pueden hacer mucho más, ya que no existen lineamientos concretos más actualizados con los cuales puedan apoyarse.

Como lo mencionan Hernández, Guadalupe \& Aguilar (2019), es importante invitar a las escuelas a considerar las prácticas de EA como herramientas de aprendizaje y generación de un pensamiento crítico en los estudiantes, más allá de que su fin sea mantener limpio el lugar o ahorrar agua, la meta podría ser que auxiliaran en el desarrollo del niño y en la labor de los docentes.

Cuando se aplica la EA en diversas actividades, se pueden obtener beneficios en la formación de los estudiantes, como aprender a trabajar en equipo, reforzar valores como la solidaridad, el compañerismo, la disciplina, la puntualidad, el amor por la naturaleza, así como también vincular el contenido teórico con la vida diaria (Espejel \& Castillo, 2015).

Desde el colectivo, surge la necesidad representada en una EA importante pero desconocida, la necesidad de hacerla propia e integrarla para desarrollar un procesos de concientización individual y social para la construcción de nuevos escenarios.

\section{Discusión}

En esta sección se presenta la discusión científica que emerge de los resultados del estudio. Se analiza categoría por categoría, los principales resultados a la luz de la literatura internacional en el área, con la interpretación de los mapas semánticos. En primer lugar, conocimiento de la EA, los profesores valoran este tipo de disciplina, pero manifiestan desconocimiento de ella. Corrales (2010) en su estudio de trabajar la EA desde la Educación Física manifiesta que es un proceso continuo, que busca formar y modificar las actitudes de las personas, por lo cual da importancia a esta materia e incita al profesorado a formarse en esta área. Por otra parte, Cañabate, Tesouro, Puigggali y Zagalaz (2019) mencionan que la EF con actividades innovadoras y adaptadas a un contexto puede ser beneficioso practicar la actividad física en entornos naturales, aumentando la conciencia por su cuidado.

Por otra parte, la representación de los estudiantes distingue la conciencia que ellos tienen sobre el cuidado del ambiente, pero poco conocimiento sobre el contenido y formas de cuidado este. Mora, (2013) expresa que la base del conocimiento de los estudiantes es determinante para el futuro, por lo que es importante que estos tengan conocimiento y conciencia sobre la importancia del cuidado y conservación del ambiente, es por ello que los establecimientos educacionales deberían ofrecer alternativas a las Bases Curriculares a través de talleres de EA en horario no lectivo.

En cuanto a la categoría EA y currículum, debido al desconocimiento de los profesores sobre la EA y poco apoyo por parte del Mineduc para generar contenidos o sugerencias, causan poco desarrollo de la EA en clases de Educación Física, por lo que los profesores solo lo indican como un objetivo transversal. Torres et al. (2017) mencionan que un profesor que tenga conocimientos y actitudes ambientales generará estudiantes más conscientes con el medio ambiente (Tuncer et al, 2009, citado por Torres et al, 2017). El profesor es el mediador entre lo que se diseña en el currículum y el aprendizaje al igual que en la adquisición de conciencia ambiental por parte de los estudiantes. En este sentido, el conocimiento pedagógico es importante por sobre el disciplinar para poder planear, organizar y ejecutar situaciones de enseñanza-aprendizaje de calidad (Almoacid-Fierro, Merellano-Navarro, Feu, Vizuete y Orellana, 2019).

En relación con los estudiantes, tienen conciencia de la importancia de la EA, para el desarrollo de actitudes positivas, formación de hábitos y cuidado de la naturaleza, además de mencionar algunas actividades que se realizan en sus colegios, pero de manera irregular, por lo que sugieren una conexión de EA con la Educación Física de manera constante durante su desarrollo escolar. La Ley N²0911/2016 de Formación Ciudadana contempla un plan de formación ciudadana en establecimientos para el desarrollo social e integral de los estudiantes (MMA, 2018). Sin embargo, según Baños y Arrayales (2019) para que los estudiantes se sientan motivados, debe existir conocimiento de las estrategias a implementar y que estas sean sesiones con un clima de aprendizaje adecuado para el disfrute y así evitar el clima de evitación-rendimiento.

En relación con la categoría estrategias de enseñanza de los profesores, éstos promueven al cuidado y preservación del ambiente, pero desde una mirada personal o de decisiones de los establecimientos con baja disposición al cambio por lo que es redundante y poco llamativo para los estudiantes. Castillo-Retamal \& Cordero-Tapia (2019) mencionan el privilegio de la Educación Física al poder desarrollarse desde la práctica y vivencia, por lo que ayuda a que los aprendizajes sean significativos. 
Finalmente, en relación con la categoría cuidado del medio ambiente, los estudiantes plantean que están conscientes de la contaminación y admiten que practican hábitos que les enseñan en casa, por lo que proponen una implementación que abarque contenidos de la EA en la Educación Física. Así como lo mencionan Hernández et al. (2019), señalando que la práctica de la EA genera un pensamiento crítico en los estudiantes y ayuda al desarrollo de ellos como también a cumplir con la labor de los profesores.

Al igual que Espejel \& Castillo (2015), cuando mencionan que, al vincular contenido teórico con la vida diaria, y al aplicarla, la EA en diversas actividades educativas y recreativas, que traen beneficios valóricos como el compañerismo, disciplina, puntualidad y el liderazgo (Carreño, Díaz, López y Martín, 2019).

\section{Conclusión}

En relación con el objetivo general del estudio «Describir y comprender en profundidad las representaciones sociales de los estudiantes y profesores de Educación Física sobre Educación Ambiental de los colegios Armando Dufey y Greenhouse de la comuna de Temuco» se concluye que, la EA es un área que se debe presentar, enseñar y tratar en los distintos establecimientos educacionales de la región de la Araucanía, sin importar que estos sean de tipo municipal o particular. La EA es un tema que nos compete a todos como seres humanos, ya que consiste en el conocimiento, cuidado y preservación de nuestro entorno natural y el medioambiente, es por esto por lo que nos parece fundamental y de especial relevancia el que se dé a conocer desde temprana edad en el aula, para que así, niños, niñas y adolescentes puedan crecer comprendiendo la relevancia de cuidar el medio en el que vivimos y todos lo que esto implica.

Mediante los instrumentos aplicados a ambos establecimientos, se logra interpretar que no se abarca la EA como se espera en las distintas asignaturas que se podría tratar, como, por ejemplo: Educación Física y Ciencias Naturales. Sin embargo, los profesores mencionan en las entrevistas aplicadas, que de acuerdo con las Bases curriculares realizan actividades al aire libre, a pesar de que no sean con mucha frecuencia. En cuanto a los estudiantes se puede deducir que son conscientes de la falta de la EA en sus asignaturas, pero de igual manera vinculan algunas actividades que realizan en la cotidianidad, como realizar actividad física al aire libre o visitar entornos naturales cuidando y protegiendo estos.
En relación con los objetivos específicos planteados en esta investigación, se dan las siguientes conclusiones. A partir del primer objetivo específico «Identificar que entienden por Educación Ambiental los estudiantes y profesores de Educación Física de los colegios Armando Dufey y Greenhouse de la comuna de Temuco» se concluye que, los docentes y estudiantes demuestran una valoración positiva por la EA, pero en su gran mayoría, los alumnos y profesores no tienen un concepto claro y concreto de qué es la EA y por ende tampoco comprenden qué significa y cuáles son los ámbitos que componen ésta.

De acuerdo con el segundo objetivo específico «Describir cómo aplican la Educación Ambiental a través del currículum los profesores de Educación Física de los colegios Armando Dufey y Greenhouse de la comuna de Temuco». Al igual que con el primer objetivo previamente expuesto, los profesores demostraron no tener un concepto claro sobre EA y manifestaron que no la aplican formalmente, utilizando alguna metodología o estrategia en particular, sino que la imparten basados en conocimientos propios y sugerencias del establecimiento para desarrollar la EA desde un área en particular, además declaran discursivamente, las pocas herramientas que tiene el Mineduc para apoyar a los docentes curricularmente en esta materia.

Abordando el tercer objetivo específico, «Identificar el cuidado que los escolares le dan a la naturaleza en relación con la contaminación». Los estudiantes expresaron que la contaminación es una de las áreas de la EA sobre la cual poseen mayor conocimiento y es básicamente a la que más relevancia le dan, puesto que su idea de EA se reduce al cuidado del planeta, protegiendo el entorno natural, los seres vivos que a él pertenecen y mermando la cantidad de basura que los seres humanos desechamos.

En consecuencia, y observando toda la evidencia empírica que ha sido puesta a disposición mediante este documento, se puede inferir que los estudiantes y profesores, no poseen un concepto claro respecto a la EA, todas las ramas qué esta posee y los diversos puntos de vista desde los que puede ser abordada, ya que desde su base en el ámbito educativo, tampoco existe claridad acerca de los contenidos que los docentes deben impartir en cada nivel escolar y por consiguiente, los estudiantes mucho menos tendrán claridad de estos contenidos. Sin embargo, las representaciones sociales de los docentes y estudiantes revelan un interés por el tema y expresan diversas opiniones e ideas respecto de cómo podría ser abordado el contenido, de manera que el conocimiento sobre EA crezca y se desarrolle en el ámbi- 
to escolar, promoviendo el cuidado, preservación y respeto por nuestro planeta y todos los seres que lo componen.

\section{Referencias}

Almonacid-Fierro, A., Merellano-Navarro, E., Feu Molina, S., Vizuete Carrizosa, M., \& Orellana Fernández, R. (2019). Perspectiva cualitativa en la construcción del conocimiento didáctico del contenido del profesorado de Educación Física (Qualitative perspective in the construction of didactic content knowledge in Physical Education teachers). Retos, 36, 459-468. https:/ / doi.org/10.47197/retos.v36i36.68840

Amezcua, M. \& Gálvez, A. (2002). Los modos de análisis en investigación cualitativa en salud: perspectiva crítica y reflexiones en voz alta. Revista Española de Salud Pública, 76 (5). issn: 1135-5727. Recuperado de: https:// www.redalyc.org/articulo.oa?id=17076505

Arriagada, C. \& Fuentes, G. (2021). Estudio de casos y teoría fundamentada como desafío y oportunidad para la investigación educativa en la escuela. En San Martín, D. Investigación educativa desde la escuela propuestas y desafíos (pp.168-205). CIED-UCT. Recuperado de: https: / / www.researchgate.net/publication /

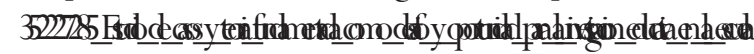

Bryman, A. (2016). Métodos de investigación social . Prensa de la Universidad de Oxford. Recuperado de: https:/ / $\mathrm{s}$ c h o l a r. g o o g l e . c o m /

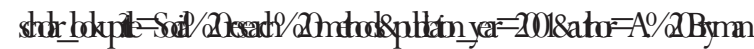

Baena, A. \& Granero, A. (2008). Las actividades físicas en la naturaleza en el currículum actual: contribución a la educación para la ciudadanía y los derechos humanos. RETOS. Nuevas Tendencias en Educación Física, Deporte y Recreación, (14), 48-53. [Fecha de Consulta 22 de Julio de 2021]. issn: 1579-1726. Disponible en: https: / / www.redalyc.org/articulo.oa?id=345732279008

Ekins, P., Gupta, J. \& Boileau, P. (2019). Global eviroment OutlookGEO-6: Healthy Planet, Healthy people(Edition $\mathrm{N}^{\circ} 6$ ). PaulEkins.https://www.unep.org/es/resources/perspectivas-del-medio-ambiente-mundial-6

Hernández, C., Guadalupe, L. \& Aguilar, R. (2019). Prácticas escolares de educación ambiental en Tecate, Baja California. Región y sociedad, 31, e1150. https:// dx.doi.org/10.22198/rys2019/31/1150

Cañabate Ortíz, D., Tesouro Cid., M., Puigggali Allepuz, J., \& Zagalaz Sánchez, M. L. (2018). Estado actual de la Educación Física desde el punto de vista del profesorado. Propuestas de mejora. (Current state of Physical Education from the point of view of teachers.
Improvement proposals). Retos, 35, 47-53. https: / / doi.org/10.47197/retos.v0i35.63038

Carreño Cardozo, J., Díaz Velasco, A., López Domínguez, S., \& Martín Reyes, J. (2019). ¿Qué se investiga en formación docente en educación física y en recreación? (What is researched in teacher training in physical education and recreation?). Retos, 36, 3-8. https: // doi.org/10.47197/retos.v36i36.51456

Castillo-Retamal, F., \& Cordero-Tapia, F. (2019). La educación ambiental en la formación de profesores en Chile. UC Maule, (56), pp. 9-28. Recuperado de: https:/ /doi.org/10.29035/ucmaule.56.9.Talca, Chile.

Corrales, A. (2010). Trabajar la Educación Ambiental desde la Educación Física. Revista educativa digital, 3 (5), 45-64. Recuperado de: https://drive.google.com/file/d/ 1qn60m9VxEv2FgC25lIM-HScSAhLFdGW9/view

Creswell, J. (2014). Research design: Qualitative, quantitative, and mixed methods approaches. Thousand Oaks, CA: SAGE Publications. Recuperado de: https:/ /epedagogium.upol.cz/pdfs/epd/2016/04/08.pdf

Delors, J. (1996). de la publicación: La Educación Encierra un Tesoro. Informe a la UNESCO de la Comisión Internacional sobre la Educación para el Siglo XXI. Laurus, 14(26), 136-167. Recuperado de: http:/ /www.cca.org.mx/ apoyos/competencias/m4/Delors+J(1996)9-27.pdf

Espejel, A., \& Castillo, I. (2019). Educación ambiental en el bachillerato: De la escuela a la familia. Alteridad, 14(2), 231-242. https:/ /doi.org/10.17163/alt.v14n2.2019.07 Torres, L., Benavides, J., Latoja, C., \& Novoa, E. (2017). Presencia de una Educación Ambiental basada en conocimiento, actitudes y prácticas en la enseñanza de las ciencias naturales en establecimientos municipales de la ciudad de Los Ángeles, Chile. Estudios pedagógicos (Valdivia), 43(3), 311-323. Recuperado de: https: / / doi.org/10.4067/s0718-07052017000300018

Gómez-Lee, M. (2019). Agenda 2030 de desarrollo sostenible: comunidad epistémica de los límites planetarios y cambio climático. Opera, 24, 69-93. DOI: https: / / doi.org/10.18601/16578651.n24.05

Ministerio del MedioAmbiente.(2018). Educación ambiental para la sustentabilidad: síntesis para el docente. Recuperado de https: / / mma.gob.cl/wp-content/uploads/ 2018/08/Guia-Docentes-EA_web.pdf

Ministerio de Educación. (2013). Cómo llegamos a ser una comunidad Educativa Sustentable, Educación para el Desarrollo Sustentable. Santiago, Chile: Ministerio de Educación. Recuperado de: https:// bibliotecadigital.mineduc.cl/bitstream/handle/ $20.500 .12365 / 585 / \mathrm{M} \mathrm{O} \mathrm{N} \mathrm{O} \mathrm{-}$ 499.pdf?sequence $=1$ \&isAllowed $=\mathrm{y}$ 
Mora, W. (2013). Educación en Ciencias y Educación Ambiental: Necesidad de una relación mutuamente beneficiosa. Revista EDUCyT, 134-14. Recuperado de: https: / / www.researchgate.net / publication /

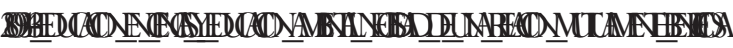

Ministerio del MedioAmbiente.(2018). Educación ambiental: Una mirada desde la institucionalidad ambiental chilena. Recuperado de https: / /educacion.mma.gob.cl/ wp-content/uploads/2018/09/LBRO-EDUCACIONAMBIENTAL-final_web.pdf

Moscovici, S. (1979). El psicoanálisis su imagen y su público. Buenos Aires: Editorial Huemul. Recuperado de: https: / /taniars.files.wordpress.com/2008/02/moscovici-elpsicoanalisis-su-imagen-y-su-publico.pdf

Moscovici, S. (1988). Notes towards a description of social representations. European Journal of Social Psychology, 18, 211-250. Recuperado de: https:// www.researchgate.net / publication / 22778646_Notes_Towards_a_Description_ of_Social_Representations

Moscovici, R. (2012). Influencia minoritaria. Psicología social: revisitando los estudios clásicos, 91. Recuperado de: h t t p s : / / b o o k s . g o o g l e . c l /

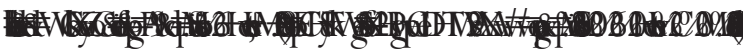

López, V., \& López, E. (1997). Tratamiento de la educación ambiental desde el área de educación física. Problemática y propuestas de acción. Apunts. Educación física y deportes., 4(50), pp. 76-83. Recuperado de https:// www.raco.cat/index.php/ApuntsEFD/article/view/ 308564

Tracy, S. (2017). Aportes pedagógicos a la educación ambiental: una perspectiva teórica. Revista de investigaciones Altoandinas., 20(3), 333-346. Recuperado de http:// w w w . $\mathrm{s}$ c i e l o . o r g . p e / scielo.php?script $=$ sci_arttext\&pid $=$ S2313$29572018000300007 \& \operatorname{lng}=$ es\&nrm $=$ iso\&tlng $=$ es

Díaz, P. (2020). El abuso sexual infantil en el entramado de las representaciones sociales de la niña. Trabajo social, 22 (1), 127-151. Recuperado de: https:// dx.doi.org/10.15446/ts.v22n1.79237

Corrales, A. (2010). Trabajar la Educación Ambiental desde la Educación Física. Revista educativa digital, 3 (5), 45-64. Recuperado de: https://drive.google.com/file/d/ 1qn60m9VxEv2FgC25lIM-HScSAhLFdGW9/view

Sureda-Negre, J., Oliver-Trobat, M., Catalan-Fernández, A., \& Comas-Forgas, R. (2014). Environmental education for sustainability in the curriculum of primary teacher training in Spain. International Research in Geographical and Environmental Education, 23(4), 281-293. https://doi.org/10.1080/10382046.2014.946322

Yeh, S.-S., Ma, T., \& Huan, T.-C. (2016). Fomento del emprendimiento social para la industria hotelera mediante la promoción de la educación ambiental. International Journal of Contemporary Hospitality Management, 28(6), 1204-1224. Recuperadode: https:/ /doi.org/10.1108/ijchm-03-2014-0122

Taylor, S. J., \& Bogdan, R. (1987). Introducción a los métodos cualitativos de investigación (Vol. 1). Barcelona: Paidós. Recuperado de: http:/ /mastor.cl/blog/wp-content/ uploads/2011/12/Introduccion-a-metodos-cualitativosdeinvestigaci $\% \mathrm{C} 3 \% \mathrm{~B} 3 n$-Taylor-y-Bogdan.-344-pagspdf.pdf

Torres Rivera, Laura Beatriz, Benavides Peña, Juan Eloy, LatojaVollouta, Catalina José y Novoa Contreras, Elissette Rafaela. (2017). Presencia de una educación ambiental basada en conocimientos, actitudes y prácticas en la enseñanza de las ciencias naturales en establecimientos municipales de Los Ángeles, Chile. Estudios pedagógicos (Valdivia) , 43 (3), 311-323. https: / /dx.doi.org/ 10.4067/S0718-07052017000300018

Pulido, V., \& Olivera, E. (2018). Aportes pedagógicos a la educación ambiental: una perspectiva teórica. Revista de Investigaciones Altoandinas, 20(3), 333-346. https:/ / dx.doi.org/10.18271/ria.2018.397

UNESCO. (2006). Proyecto de Plan de Aplicación Internacional del Decenio de las Naciones Unidas de la Educación para el Desarrollo Sostenible. París, Francia. Recuperado de: http:/ / w w w. la c u l t. un e s co.org / d o c c / 2012_Educ_para_des_sost.pdf

Gibbs, G. (2012). El análisis de datos cualitativos en investigación cualitativa. Madrid: Ediciones Morata.

San Martín, D. (2014). Teoría fundamentada y Atlas.ti: recursos metodológicos para la investigación educativa. Revista electrónica de investigación educativa, 16(1), 104-122. Recuperado de: http:// w w w. $\mathrm{s}$ c i e l o . o r g. $\mathrm{m}$ x /

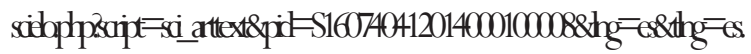
Stake, R. (2015). Estudios de Caso Cualitativo. En: N. Densin, yY. Lincoln (Coords.). (2013), Manual de Investigación Cualitativa.Vol. III: Las Estrategias de Investigación Cualitativa. pp. 154-197. Barcelona, España: Gedisa.

Strauss, A. \& Corbin, J. (2002). Bases de la investigación cualitativa: Técnicas y procedimientos para desarrollar la teoría fundamentada. Medellín, Colombia: Universidad de Antioquia. 